\title{
Wavelet Analysis for Brain-Function Imaging
}

\author{
René A. Carmona, Member, IEEE, Wen L. Hwang, and Ron D. Frostig
}

\begin{abstract}
The purpose of this paper is to present a new algorithmic procedure for the analysis of brain images. This procedure is specifically designed to image the activity and functional organization of the brain. Our results are tested on data collected and previously analyzed with the technique known as in vivo optical imaging of intrinsic signals. Our procedure enhances the applicability of this technique and facilitates the extension of the underlying ideas to other imaging problems (e.g., functional MRI). The thrust of the paper is two fold. First, we give a systematic method to control the blood vessel artifacts which typically reduce the dynamic range of the image. We propose a mathematical model for the vibrations in time of the veins and arteries and we design a new method for cleaning the images of the vessels with the highest time variations. This procedure is based on the analysis of the singularities of the images. The use of wavelet transform is of crucial importance in characterizing the singularities and reconstructing appropriate versions of the original images. The second important component of our work is the analysis of the time evolution of the fine structure of the images. We show that, once the images have been cleaned of the blood vessel vibrations/variations, the principal component of the time evolutions of the signals is due to the functional activity following the stimuli. The part of the brain where this function takes place can be localized and delineated with precision.
\end{abstract}

\section{INTRODUCTION AND NOTATIONS}

$\mathbf{O}$ PTICAL imaging of intrinsic signals is a recently developed in vivo imaging technique that enables the visualization of the functional organization of the brain with the highest spatial resolution $(\approx 50 \mu \mathrm{m})$ compared with other in vivo brain imaging techniques (see [8], [9], and [18]). The principle behind this imaging technique is that when the brain is illuminated, active areas reflect less light compared to nonactive areas, and that the stronger the activity, the smaller the reflectance. The change in reflectance after brain activation has a characteristic evolution in time and is termed intrinsic signal. Activity-dependent intrinsic signals are small $\left(10^{-4}\right.$ to $10^{-3}$ of the illumination) and slow (rise time to peak on the order of 1-2 s) relative to the time of action-potentials in the brain. Intrinsic signal's imaging, however, produces detailed highresolution in vivo functional maps from large areas of the brain that are identical to the areas of spiking neurons in the brain.

Manuscript received October 5, 1994; revised June 2, 1995. The work of $\mathbf{R}$. A. Carmona was supported in part by ONR N00014-91-1010, and the work of W. L. Hwang was supported by NSF IBN 9405146. The Associate Editor responsible for coordinating the review of this paper and recommending its publication was C. Roux.

R. A. Carmona was with the Department of Mathematics, University of California at Irvine, Irvine, CA 92717 USA. He is now with the Department of Civil Engineering and Operations Research, Princeton University, Princeton, NJ 08544 USA; e-mail: rcarmona@princeton.edu.

W. L. Hwang was with the Department of Mathematics, University of California at Irvine, Irvine, CA 92717 USA. He is now with the Institute of Information Science, Academia Sinica, Taipei, Taiwan.

R. D. Frostig is with the Department of Psychobiology, University of California at Irvine, Irvine, CA 92717 USA.

IEEE Log Number 9413897.
Using optical imaging of intrinsic signals, a new functional organization of the visual cortex has been discovered (see [3]) and for the first time, the relations between several functional systems within the visual cortex could be investigated (see [1]). Recently, further development of the technique enabled this imaging to be obtained in a noninvasive way to the brain (see [14]) and thus led, for the first time, to the ability to image the functional organization of the brain repeatedly from the same animal over time (a chronic preparation) (see [15]).

The major sources underlying intrinsic signals are: 1) changes in blood volume in an active area of the brain; 2) changes in the oxygenation level of hemoglobin in active areas of the brain; and 3) changes in the volume of active neurons (see [8]). While all three mechanisms are active simultaneously after brain activation, illumination of the brain with specific narrow wavelengths highlights the dominance of one specific mechanism. Most experiments are performed with 630-nm illumination that highlights the blood oxygenation mechanism. As a consequence, blood vessels in the imaged area tend to produce strong signals that reduce the dynamic range of the images, and hence the quality of the images. Blood vessel artifacts are the strongest source of noise in the system and, in extreme cases, completely mask the visualization of brain activation. Therefore, the reduction of their contribution is the single most important step to obtain better images of brain activity. Similar problems are encountered with functional Magnetic Resonance Imaging (fMRI) since this imaging technique is also based on measuring the oxygenation level of the blood [2], [11], [16]. Thus, the major priority for better functional imaging is to clean the process from the bloodvessel artifacts without reducing the visualization of brain activity. This manuscript describes the first successful attempt to solve this problem by developing an algorithmic procedure for functional imaging. It is based on a new mathematical model for the evolution of the light reflectance, and the treatment of the images is based on the features of this model. We tested our algorithm on data previously processed with various ad hoc division methods tailored to the experiments (see, for example, [1], [3], [8], [9], [14], [15,] and [18] for details on these diverse imaging procedures). In all cases, the performance of our single algorithm was at least as good.

One of the driving forces of our study was the potential applicability of our automatic imaging technique to fMRI problems (see [11] and [16] for early work in this area and the discussion in [5] for the use of our algorithm for long sequences of images). Nevertheless, it is important to emphasize that the nature of the data (and consequently the approach used in the analysis) is very different. Indeed, most fMRI studies require long sequences of images (typically between 40 and 140) in order for the stimuli to be presented 
many times in a periodic manner. The responses to the stimuli are also periodic in time and hence can be picked up by fitting a periodic waveform to the response as in [6], or by the use of standard time-frequency techniques as, for example, in [2] or [10]. The shortcomings of these approaches and the advantages offered by our algorithm for brain imaging are documented in [5]. Since we are working from data containing only one occurence of the stimulus, the response is localized in time (like a transient) and Fourier techniques cannot be used to detect the activity resulting from the stimulus. Also, the resolution of our images is much finer than the resolution of images produced by fMRI (and PET). This is another source of difficulty since the experimental noise and the vibrations of the blood vessels increase the dynamic ranges of the images and significantly lower the signal-to-noise ratios.

The thrust of the paper is twofold. First, we give a systematic method to reduce the blood vessel artifacts. We propose a mathematical model for the vibrations in time of the main veins and arteries and we design a new method for cleaning the images of the vessels with the highest variations in time. This procedure is based on the analysis of the singularities of the images. We use wavelet transform to characterize these singularities and to reconstruct appropriate versions of the original images. The second important component of our algorithm is the analysis of the time evolutions of the reflectance values of the pixels forming the images. The fine analysis of these evolutions, including more wavelet transforms and statistical data analysis processing, leads to the identification of the response area of the brain to the stimuli. Our mathematical model implies that, in the absence of blood vessel artifacts, the covariance matrix of the light reflectance time evolutions has rank one. The eigenvector corresponding to the only nonzero eigenvalue gives the intrinsic signal while the projections onto this eigenvector form an image of the response of the brain to the stimulus. Since we cannot expect a perfect cleaning of the images, a perturbation argument is necessary to justify our search for the largest eigenvalue (and the corresponding eigenvector) of the time evolution covariance matrix. This is our rationale for the use of the singular value decomposition of matrices. In some sense, this is a simple form of principal component analysis. It is important to emphasize that our use of this technique is different from previous attempts to use it to image brain functions as in, for example, [7] and [17]. We are classifying pixel time evolutions instead of images. The works of [7] and [17] are plagued with difficulties created by the high dimensionality of the vectors to classify: each observation is an image, so its dimension is the number of pixels and the resulting covariance matrix is very large. As a consequence, the computational burden is the main obstruction to the implementation of the method and most of the efforts of [7] and [17] are devoted to finding solutions for the dimensionality problem. By contrast, we use a form of principal component analysis in a situation with a large number of observations (as many as pixels in a frame) of low dimension (typically between 7 and 9) and the computations are very fast.

The correctness of the specific boundaries given by these image manipulations has been compared with the results of

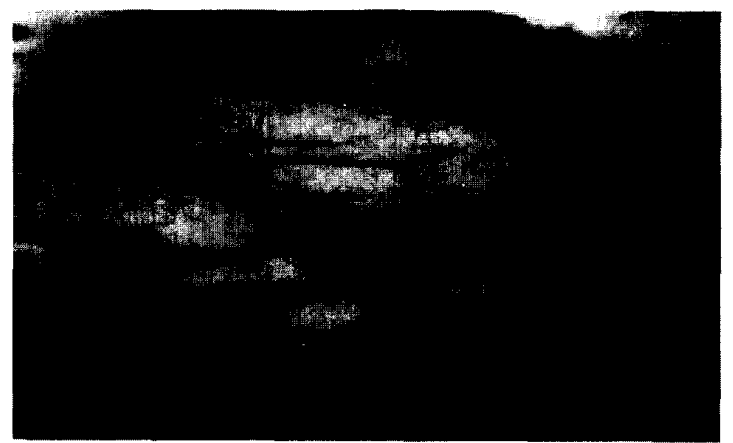

Fig. 1. Image of the somatosensory cortex of a rat. A $6.7 \times 5.0$-mm region of the cortex is shown. It is illuminated with green light $(540 \mathrm{~nm})$ to enhance the visualization of the blood vessels.

[14] using structural and functional markers. In order to illustrate our method, we reproduce the results obtained in three experiments. More experimental results can be found in [5].

We refer to [14] for a description of the main features of the in vivo imaging technique and for details of the experimental protocols. We shall only describe the spatial and temporal features of the intrinsic signals in somatosensory cortex which are relevant to the understanding of the algorithms which we introduce.

For the purpose of the present discussion, we concentrate on experiments producing a sequence of images of the brain taken by a slow scan scientific CCD camera. Each image has $n_{r}=144$ rows and $n_{c}=192$ columns of reflectance values. The value of each pixel is a grey level coded by an integer between 0 and 255. Typical experiments produce sets of $n_{f}=9$ or $n_{f}=10$ successive images (also called frames) each lasting $500 \mathrm{~ms}$. The stimulus is applied between the second and the fifth frame and the relaxation process covers the remaining images. Fig. 1 shows a typical image obtained during the experiments. Any of the $n_{f}$ images taken from the same experiment would be visually indistinguishable from this particular one. It is important to emphasize that the main hindrance to the analysis of these images is the difference in scale between the time variations of the blood vessels and the variations of the region of activity.

\section{The Mathematical Model}

The mathematical model is as follows. We use the notation $x$ for the row number and $y$ for the column number of a pixel. We use the notation $t$ for the image number. $x$ varies from 1 to $n_{r}=144, y$ varies from 1 to $n_{c}=192$, while $t$ varies from 1 to $n_{f}=10$ or $n_{f}=9$, depending on the experimental conditions. Let us denote by $f(t, x, y)$ the reflectance intensity at the pixel $(x, y)$ of the $t$ th image. We assume that it is of the form

$$
f(t, x, y)=I(t, x, y)+V(t, x, y) .
$$

The function $I(t, x, y)$, when regarded as a function of $t$, gives the evolution over time of the intrinsic signal at the location $(x, y)$. The function $V(t, x, y)$ represents the large vibrations/variations of the reflectance values at time $t$ and at the pixel location $(x, y)$. As explained in Section $\mathbf{I}$, we are 


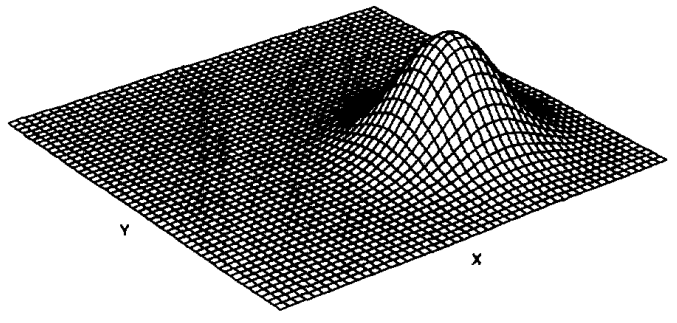

(a)

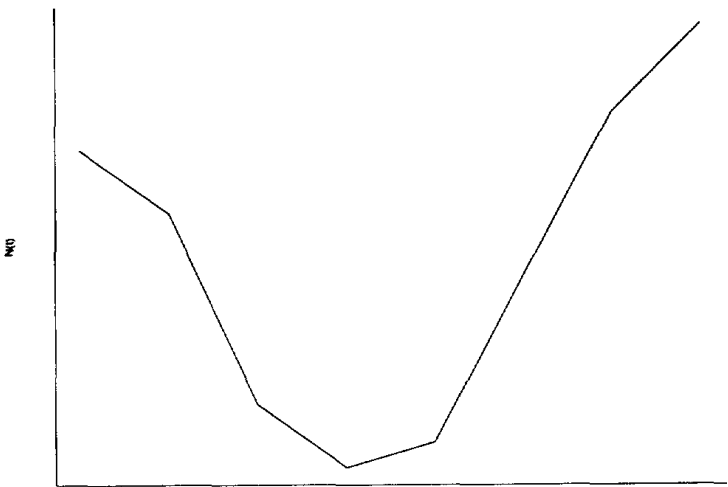

(b)

Fig. 2. (a) Surface plot of the function $S(x, y)$ taming off the intrinsic signal away from the activity region. (b) Graph of the function $N(t)$ used as a model for the typical time evolution of the response of the pixels in the active region. Note that this plot is always given "upside down" in publications on the subject.

interested in the estimation of the intrinsic signals despite the presence of the component $V(t, x, y)$. Indeed, these signals contain the characteristics of the responses of the various parts of the brain to the stimuli. We assume that the function $I(t, x, y)$ is the sum of a baseline constant $E(I)$ and a variation term in the form of a tensor product. More precisely, we assume that the intrinsic signals are of the form

$$
I(t, x, y)=E(I)+S(x, y) N(t)
$$

where $S(x, y)$ is a function of the pixel location only and $N(t)$ is a function of time. The baseline constant $E(I)$ can be viewed as a mean signal and computed as the time average of the intrinsic signal $I(t, x, y)$. As such, its value depends only upon the location $(x, y)$ of the pixel. For the sake of simplicity, we assume further that it is independent of the pixel.

The function $S(x, y)$ can be thought of as a smooth bellshaped surface centered around the center of the activity region where the response to the stimulus takes place. It should decay to zero away from the center of this activity region. See Fig. 2(a) for the plot of the function $S(x, y)$. The function $N(t)$ gives the typical variations (around the value of the baseline constant) of the time evolution of the intensity at the pixels of the activity region. Fig. 2(b) shows how the graph of such a time evolution appears. Slow decay in the initial stage accentuated decay followed by a sharp turn around the mid-life time of the signal and rapid increase leading to a clear overshoot above the original baseline constant. Note that, for the sake of clarity of the mathematical derivation of the model, we break away from the common practice of plotting the intrinsic signals upside down, as in [14], for example.

It is not easy to explain the biological nature of the relaxation effect and the overshoot, but it is relatively easy to give an attractive rationale to our mathematical model. For the purpose of the present discussion, we shall take some freedom with the rigor of a detailed biological explanation and use instead a more picturesque (obviously naive) justification for the choices of the functions $S(x, y)$ and $N(t)$. The biological response to the stimulus is localized in a region which, because of a sudden increase in the level of activity, requires an increase in the blood supply. For a short time, the center of the region can be viewed as a "blood sink." The topology of this sink is given by the bell-shaped surface defined by the time independent function $S(x, y)$. Because of the change in blood volume and the deoxygenation process, the incoming light is absorbed more strongly and this causes the dip in the graph of the time evolution exemplified by the function $N(t)$. This explanation is confirmed by the experimental results which we give in Section V.

\section{A. Time Evolutions and Vibration Maps}

The time evolution of the images is best studied via the analysis of the time derivatives

$$
f_{t}(x, y)=\frac{\partial f(t, x, y)}{\partial t}
$$

computed at each pixel $(x, y)$. Note that

$$
f_{t}(x, y)=\frac{\partial I(t, x, y)}{\partial t}+\frac{\partial V(t, x, y)}{\partial t} .
$$

This time derivative behaves differently when the pixel $(x, y)$ is near an active blood vessel. Indeed, in this case, the second term overwhelms the first term and makes it extremely difficult (if not impossible) to see the activity as exemplified by the intrinsic signals. Previous measurements (see [14]) have shown that the dynamic range of the intrinsic signals is about -30 $\mathrm{dB}$. It is argued in [14] that

$$
\left|\frac{\partial I(t, x, y)}{\partial t}\right| \approx 10^{-3}\left|f_{t}(x, y)\right|
$$

at the peak of the time evolution of the pixels $(x, y)$ in the active region where the response to the stimulus can be detected. It is clear from this fact that the presence of the intrinsic signals in the active region will not be detected if the main source of variations (given by $V(t, x, y)$ in our model) is not controlled. The function $\partial I(t, x, y) / \partial t$ is, by definition, a bounded flat function essentially equal to zero away from the active region, and we expect that, especially when the pixel $(x, y)$ is near or on a blood vessel, the variations will mostly come from the term $V(t, x, y)$. If we denote by $\nabla f(t, x, y)$ the gradient with respect to the space variables $x$ and $y$, i.e., if we set

$$
\nabla f(t, x, y)=\left[\frac{\partial f(t, x, y)}{\partial x}, \frac{\partial f(t, x, y)}{\partial y}\right]
$$

then we can use the square of the length of this vector, i.e., 


$$
\|\nabla f(t, x, y)\|^{2}=\left|\frac{\partial f(t, x, y)}{\partial x}\right|^{2}+\left|\frac{\partial f(t, x, y)}{\partial y}\right|^{2}
$$

as a measure of the intensity of the gradient variations (in space) of the value of a pixel at a given time. Blood vessels have several types of variations. As time goes by, they compress and expand, and these changes lead to sharp variations in the time evolutions of their boundaries. We combine this notion of space gradient $\nabla f$ with the notion of time derivative $f_{t}$ introduced earlier to define a notion of vibration intensity with the intent to use it to discriminate the pixels responsible for the artifacts preventing the intrinsic signals from being seen. We measure these vibrations by the following quantity:

$$
[V f](x, y)=\int\left[\left|\frac{\partial f_{t}(x, y)}{\partial x}\right|^{2}+\left|\frac{\partial f_{t}(x, y)}{\partial y}\right|^{2}\right]^{1 / 2} d t
$$

We expect that this measure of the vibrations of a pixel will be large on or near the active blood vessels. This vibration measure $[V f](x, y)$ depends only upon the location of the pixel. It is different from the term $V(t, x, y)$ advocated in the introduction (1) of our mathematical model. In particular, it does not depend upon the time variable $t$.

Biological experiments have shown that the dynamic range of the blood vessel vibrations is typically larger than the dynamic range of the vibrations due to the intrinsic signals (see [14], for example). Since the vibrations of the blood vessels are the main obstruction to the identification of the intrinsic signals, it is natural to try to clean the images from these vibrations. According to the above discussion, this might be possible by identifying the regions where $[V f](x, y)$ has a large value. Identifying these regions is a first step in the cleaning of the images, the second step requiring the removal of the vibrations in these regions. We shall see how the wavelet analysis which we describe in the next section makes this enterprise possible.

\section{B. Estimation of the Intrinsic Signals}

We first assume that the removal of the large vibrations due to the blood vessels has been successfully performed. Given this assumption, the intrinsic signal $N(t)$, and most importantly the surface $S(x, y)$, can be identified from the characteristics of our model. This mathematical derivation, together with the perturbation result recalled below, form the mathematical justification of our use of the principal component analysis when the cleaning of the blood vessel artifacts has been successful.

We assume that the time variable $t$ has been sampled (i.e., that there are a finite number of images) and we denote by $t_{1}, \cdots, t_{n f}$ the times at which the images have been taken. Computing time derivatives, we have the values of the derivatives at the times $t_{1}, \cdots, t_{n_{f}-1}$. Let us denote by $\Sigma=$ $\left[\Sigma_{i, j}\right]_{i, j=1, \cdots, n_{f}-1}$ the covariance matrix of the time derivative of the intrinsic signals. A simple computation shows that

$$
\begin{aligned}
\Sigma_{i, j} & =N^{\prime}\left(t_{i}\right) N^{\prime}\left(t_{j}\right) \sigma_{S} \\
& =r^{2}\left[U U^{t}\right]_{i, j}
\end{aligned}
$$

if we use the notation $r=\tau \sigma_{S}$, and if $\sigma_{S}$ denotes the standard deviation of the response surface $S, \tau$ denotes the length of the vector of the time derivatives of the intrinsic signals

$$
\tau=\left\|\left[N^{\prime}\left(t_{i}\right)\right]_{i=1, \cdots, n_{f}-1}\right\|,
$$

and $U$ denotes the unit vector in the direction of these time derivatives, i.e.,

$$
U=\tau^{-1}\left[N^{\prime}\left(t_{i}\right)\right]_{i=1, \cdots, n_{f}-1} .
$$

The covariance matrix $\Sigma$ has rank one. In fact, up to a multiplicative factor, it is the matrix of the orthogonal projection onto the one-dimensional (1-D) subspace generated by the vector of the time derivatives of the intrinsic signal. $r^{2}$ is the only nonzero eigenvalue of the matrix $\Sigma$ and the corresponding normalized eigenvector is the vector $U$ defined above. The size of the projection of the vector $\left[\partial I\left(t_{i}, x, y\right) / \partial t\right]_{i=1, \cdots, n_{f}-1}$ onto $U$ is given by

$$
\begin{aligned}
\left\langle\frac{\partial I(\cdot, x, y)}{\partial t}, U\right\rangle & =\sum_{i=1}^{n_{f}-1} \tau^{-1} S(x, y) N^{\prime}\left(t_{i}\right) N^{\prime}\left(t_{i}\right) \\
& =\tau S(x, y) .
\end{aligned}
$$

Recall that the quantity $S(x, y)$ was introduced in the mathematical model as a time independent factor whose role was to tame the response to the stimulus away from the center of the active region. We see now that, up to the multiplicative constant $\tau$, this damping factor is given by the projection of the time derivative of the intrinsic signal onto the nontrivial eigenvector of the covariance matrix $\Sigma$. This observation will be instrumental in the algorithm which we give later to delineate the active region.

Since neither the intrinsic signal nor the covariance matrix are accessible to the experimenter, we devote the remainder of this section to derive a way to estimate them from the available data.

We now assume that most of the vibrations from the blood vessels have already been removed from the signals $f(t, x, y)$. We denote by $\tilde{f}(t, x, y)$ the cleaned signals. We have

$$
\frac{\partial \tilde{f}(t, x, y)}{\partial t}=\frac{\partial I(t, x, y)}{\partial t}+\frac{\partial \tilde{V}(t, x, y)}{\partial t} .
$$

Note that the removal of the vibrations did not affect the time derivative of the contribution of the intrinsic signals.

The term $\partial \tilde{V}(t, x, y) / \partial t$ is complex. Its presence is due to heartbeats and/or breathing of the animal, $\cdots$, and the superposition of all these contributions destroys its structure. For this reason, it is useful to view it as a noise term. Moreover, its contribution is obviously independent of the intrinsic signals. As a consequence, if we denote by $\Sigma_{\tilde{f}}$ the covariance matrix of $\partial \tilde{f}(t, x, y) / \partial t$, and by $\Sigma_{\tilde{V}}$ the covariance matrix of $\partial \tilde{V}(t, x, y) / \partial t$, we have

$$
\Sigma_{\tilde{f}}=\Sigma+\Sigma_{\tilde{V}}
$$

Since $\Sigma_{\tilde{f}}$ can be computed from the data, we try to control the perturbation $\Sigma_{\tilde{V}}$ to get information on what we are seeking, namely $\Sigma$. We use a standard result from the perturbation theory of eigenvalues of symmetric matrices (see, for example, [20]).

Lemma II-B: If $n \times n$ symmetric matrices $A$ and $B$ satisfy $A=r^{2} U U^{t}+B$ where $r>0$ and $U$ is a unit vector in $\mathbb{R}^{n}$, 
then, if we denote by $\lambda_{i}(A)$ and $\lambda_{i}(B)$ the eigenvalues of $A$ and $B$ respectively (counting multiplicity), one has

$$
\lambda_{i}(A)=m_{i} r^{2}+\lambda_{i}(B)
$$

for some nonnegative numbers $m_{1}, m_{2}, \cdots, m_{n}$, summing up to one.

This result explains the influence of the perturbation $\Sigma_{\dot{V}}$. Its effect is to smear the largest eigenvalue $r^{2}$ of $\Sigma$ and to spread it over all the eigenvalues of $\Sigma_{\tilde{f}}$ by means of the $m_{i}$ 's. A similar perturbation result holds for the corresponding eigenvectors. The modified derivatives $\partial \tilde{f}(t, x, y) / \partial t$ could be smoothed out by a low-pass filter in order to minimize the smearing effect of $\Sigma_{\tilde{V}}$ on the eigenvalue $r^{2}$ and the eigenvector $U$. This would lessen the influence of the vibration derivative $\partial \tilde{V}(t, x, y) / \partial t$. We nevertheless refrain from doing so because the experimental time series giving the time evolutions of the pixels are too short.

We use a standard "singular value decomposition" procedure to compute (or estimate) the first eigenvalue and the corresponding eigenvector of the matrix $\Sigma_{\tilde{f}} \approx \Sigma$. Since this matrix is a covariance matrix, the result of its singular value decomposition has a statistical interpretation. It is known under the name of Principal Component Analysis (PCA, for short). Even though we arrived at this procedure from a different perspective, we shall freely use the intuitive interpretations provided by this time-honored statistical point of view.

We shall denote by $\lambda_{i}$ the eigenvalues (in decreasing order) of the covariance matrix, and by $v^{(i)}$ the corresponding eigenvectors. The largest eigenvalue $\lambda_{1}$ measures the proportion of the variation which is explained by the first eigenvector $v^{(1)}$. The latter will be used as our estimation of the time derivative of the intrinsic signals, i.e., of $N^{\prime}(t)$. Finally, we shall use the size of the projection onto $v^{(1)}$ as our estimator of the function $S(x, y)$. Remember that, according to the above calculations, this gives us a map of the brain activity as a response to the stimulus.

Remark II-B: It may happen in practice that the typical time evolution velocity given by the first eigenvector $v^{(1)}$ (or any other normalized eigenvector for that matter) is the negative of the time evolution illustrated in Fig. 2; the PCA procedure searches for normalized eigenvectors of a (symmetric) covariance matrix and it cannot make the difference between a vector and its negative.

\section{Wavelet ANALySIS}

\section{A. Wavelet Transform of Images}

This section is devoted to the description of the specific wavelet transform which we use. For the sake of the present discussion, we restrict ourselves to one single image, say $\{I(x, y)\}_{(x, y)}$. Refer to [12] and [13] for details.

Let $\theta(x, y)$ be a smoothing function (low-pass filter), and let us define the two wavelets $\psi^{(1)}(x, y)$ and $\psi^{(2)}(x, y)$ by the formulas

$$
\psi^{(1)}(x, y)=\frac{\partial \theta(x, y)}{\partial x} \quad \text { and } \quad \psi^{(2)}(x, y)=\frac{\partial \theta(x, y)}{\partial y},
$$

respectively. The dyadic wavelet transform of the image $I=$ $\{I(x, y)\}_{(x, y)}$ at resolution $J$ (or equivalently at scale $s=2^{J}$ ) is defined as the set of images

$$
\left\{W_{s}^{(1)} I, W_{s}^{(2)} I\right\}_{s=2^{1}, 2^{2}, \cdots, 2^{j}} \quad \text { and } \quad S_{2^{J}} I
$$

where the wavelet transforms $W_{s}^{(1)} I$ and $W_{s}^{(2)} I$ at scale $s=$ $2^{j}$ are defined by the convolutions

$$
W_{s}^{(1)} I=I * \psi_{s}^{(1)} \quad \text { and } \quad W_{s}^{(2)}=I * \psi_{s}^{(2)},
$$

where we use the notation $\psi_{s}$ for the scaled version of $\psi$, i.e.,

$$
\psi_{s}(x, y)=\frac{1}{s^{2}} \psi\left(\frac{x}{s}, \frac{y}{s}\right)
$$

and where the so-called coarse scale component $S_{2 J} I$ is the image defined by

$$
S_{2^{J}} I=I * \varphi_{s},
$$

where $\varphi_{s}$ is the scaled version of the scaling function $\varphi$ canonically associated with $\psi$.

It will be convenient to use the following notations. For each pixel $(x, y)$, the two components $\left[W_{s}^{(1)} I\right](x, y)$ and $\left[W_{s}^{(2)} I\right](x, y)$ of the wavelet transform of the image $I$ at the scale $s=2^{j}$ can be put together into a 2-D vector $\left[W_{s} I\right](x, y)=\left[\left[W_{s}^{(1)} I\right](x, y),\left[W_{s}^{(2)} I\right](x, y)\right]$. In this way, the notation

$$
W_{s} I=\left\{\left[W_{s} I\right](x, y)\right\}_{(x, y)}
$$

stands for a vector of two images. We shall make extensive use of the modulus (or length) of this vector

$$
\left|\left[W_{s} I\right](x, y)\right|=\sqrt{\left[W_{s}^{(1)} I\right](x, y)^{2}+\left[W_{s}^{(2)} I\right](x, y)^{2}} .
$$

The name of dyadic transform is used because we are restricting the analysis to the dyadic scales $s=2^{j}$ for $j=$ $1,2, \cdots$. Let us give an enlightening interpretation of the wavelet transform as we just defined it. For each scale $s$, we first smooth the image with the low-pass filter $\theta_{s}(x, y)$, scaled according to the formula (4). This produces a blurred version of the original image, and the main features of this version are the scale $s$ characteristics of the original image. The wavelet transform $W_{s} I$ at scale $s$ is nothing but the gradient of this smoothed version of the original image. Consequently, the main features of this wavelet transform (and, in particular, of its modulus) are the points of high variations (still at the scale $s$ ) of the original image. This interpretation explains why this specific wavelet transform has been introduced and used in the analysis of singularities (see, for example, [12] and [13]).

\section{B. Reconstruction from Wavelet Transform Extremas}

We review the reconstruction of an image from the extrema of its dyadic wavelet transform. This procedure is an important element of our algorithm.

If $\{I(x, y)\}_{(x, y)}$ is an image, a very good approximation of the original image can be obtained from the mere knowledge of the extrema of its dyadic wavelet transform and from its coarse scale approximation. More precisely, if we know the locations, say $\left(x_{j, n}, y_{j, n}\right)$ of the local maxima of the modulus 

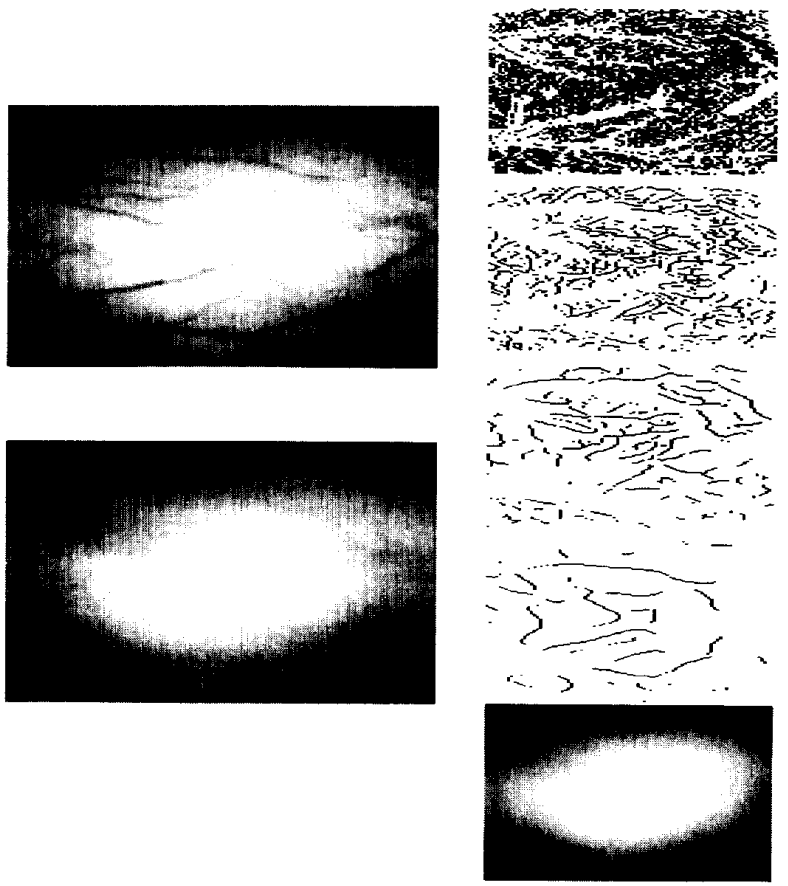

Fig. 3. Example of a partial reconstruction from a selective set of extrema of the wavelet transform. (Left) Top: A typical image. Bottom: Image reconstructed from the values of the wavelet transform at the locations of the local modulus maxima which are smaller than their $80 \%$-tiles and from the values of the coarse scale image (shown at the bottom of the right column). Notice that the blood vessels have been removed and replaced by low-frequency surfaces (compare with the original image). (Right) The scale increases from top to bottom. Positions of the local modulus maxima at the scales $s=2^{1}, 2^{2}, 2^{3}, 2^{4}$ after removing the local maxima with modulus larger than the $80 \%$-tile of the maxima sizes at each scale. Notice that the modulus maxima near the boundaries of the blood vessels are removed. The larger the value of a pixel, the brighter it shows. Bottom: Coarse scale approximation at scale $s=2^{4}$

of the wavelet transform $W_{2^{j}} I$ at scale $2^{j}$, and if we also know the corresponding values of the wavelet transform at these extrema, then it is possible to join this information to the information of the coarse scale approximation to produce an image which is very close to the original image $I$ (refer to [13] for details). Strangely enough, the beauty of this approximate reconstruction procedure is in its versatility more than in the precision of the reconstructions. Indeed, very interesting partial reconstructions are made possible by the freedom one has to trim some of the extrema and to select specific extrema before starting the reconstruction procedure. This idea has been successfully implemented in denoising problems (see, for example, [4] and [12]), but it can also be used to get rid of undesirable components of an image. This is especially easy when these components have a clear multiscale characterization in terms of some of the extrema of the wavelet transform.

Fig. 3 gives an example of application of this strategy. We present it for the sake of illustration of the present discussion. Indeed, our final form of the algorithm does not use this exact form of cleaning since it involves the trimming of extremas based both on their sizes and their locations. A typical image has been reconstructed from the extrema of its wavelet transform after discarding the extrema greater than the 80th percentile of the sizes of the extrema at each resolution. The net result is an image without the high gradients produced by the walls of the blood vessels. Such a cleaning of the images is very useful in getting rid of some of the blood vessel artifacts. We used it successfully in the analysis of several experiments. Unfortunately, it cannot control the second source of problems: the large vibrations in time of the blood vessels. A solution to this problem is obtained by including the properties of the time derivatives in the analysis.

\section{Wavelet Analysis of the Blood Vessel Artifacts}

For each scale $s$, the quadratic variation at scale $s$ is defined by

$$
\left.\left[V_{s}^{(2)} f\right](x, y)=\left.\left(\sum_{t} \mid W_{s} f_{t}\right](x, y)\right|^{2}\right)^{1 / 2} .
$$

This definition is to be understood in the following way. The wavelet transform $W_{s} f_{t}$ can be thought of as the gradient of $f_{t}$ at scale $s$. Consequently, (5) can be viewed as the implementation of (3) at the scale $s$. Notice that the time $t$ is now discrete and that integrals with respect to the variable $t$ have been replaced by discrete sums. The natural notion of quadratic variation is then obtained by summing over the scales. We get

$$
V^{(2)}(x, y)=\sum_{j=1}^{J}\left[V_{2^{j}}^{(2)} f_{t}\right](x, y) .
$$

This quantity can be used (see, nevertheless, the changes given below in the actual implementation of the algorithm) to detect the large blood vessel vibrations. The latter can be found where $V^{(2)}(x, y)$ takes large values. Once the regions of large vibrations are determined (see the white region in the image at the bottom of the right column of Fig. 4), the extrema of the wavelet transform which are located inside these regions can be trimmed. The reconstruction of such an image is shown in Fig. 5. The time evolutions of the images which are reconstructed in this way no longer contain the blood vessel vibrations.

\section{Summary}

The purpose of the wavelet analysis described above is twofold: first we make a map of the light reflectance variations/vibrations, and then we reconstruct all the images without using the extrema from the regions of high variations/vibrations. In accordance with the discussion of Section II, we denote these new signals by $\tilde{f}(t, x, y)$. According to our mathematical model, the covariance matrix $\Sigma_{\tilde{f}}$ of these time derivatives should essentially be of rank one. The (normalized) eigenvector $v^{(1)}$ corresponding to the largest eigenvalue $\lambda^{(1)}$ should be a good estimator of the derivative of the intrinsic signal archetype $N(t)$, and the image of the projections on this eigenvector should give a good estimate of the response 

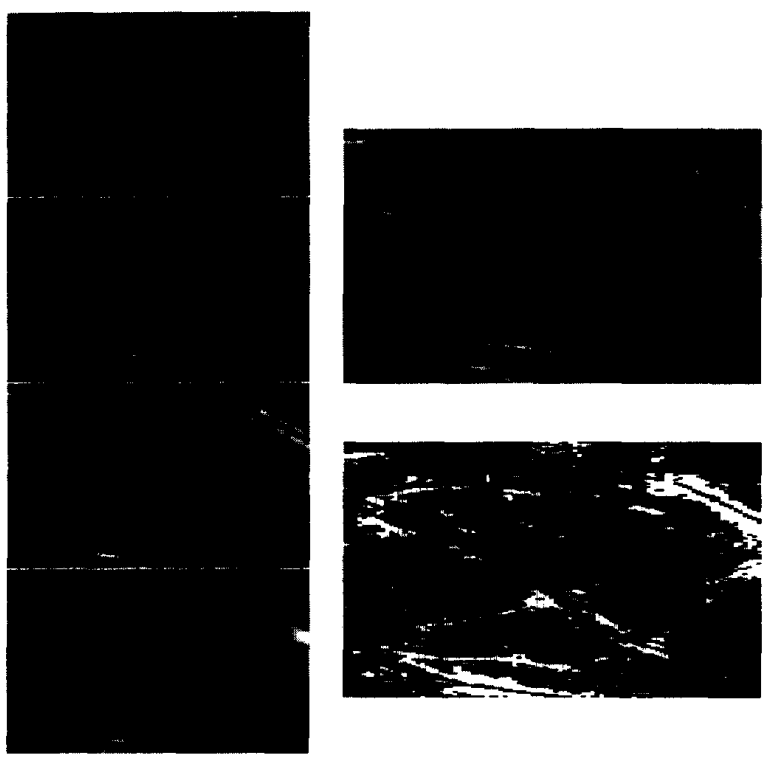

Fig. 4. Example of a vibration map. (Left) Vibrations $V_{s} f$ at the dyadic scales ranging from $s=2^{1}$ to $s=2^{4}$. The scales increase from top to bottom. As before, the larger the modulus, the brighter the pixel. (Right) Top: Total vibration $V f$. The pixels with the largest vibration energies are aggregated nearby the blood vessels. Some of these blood vessels are not visible in the original frames (see, for example, the image given at the top of the left column of Fig. 3). They form secondary structures which are captured only by the difference of adjacent time frames. Bottom: Black and white binary image showing in white the locations of the pixels where the total vibration is greater than the $90 \%$-tile of the values displayed on the top image.

surface $S(x, y)$. We are now ready to give the details of the various steps of our functional imaging algorithm.

\section{Detailed Algorithms}

\section{A. Removing the Blood Vessel Vibrations}

- We first compute, for each pixel $(x, y)$, the discrete time derivative $f_{t}(x, y)$ of the time evolution of the pixel values. This leaves us with $n_{f}-1$ images $f_{t}(1)=$ $f(1)-f(0), \cdots, f_{t}\left(n_{f}-1\right)=f\left(n_{f}\right)-f\left(n_{f}-1\right)$ of the same sizes.

- We compute, for each of these $n_{f}-1$ images, the wavelet transform $W_{s} f_{t}$ at the scales $s=2^{1}, 2^{2} ; \cdots, 2^{J}$. We use $J=4$ in the experiments reported in this paper.

- For each scale $s=2^{1}, 2^{2}, \cdots, 2^{J}$, we compute the variation $V_{s} f$ at scale $s$. According to the discussion of the previous section, this is the image whose value at pixel $(x, y)$ gives the total variation (over time) of the values of the modulus of the wavelet transforms $\left[W_{s} f_{t}\right](x, y)$ at scale $s$ of the time derivative $f_{t}$ at time $t$. Instead of considering the square root of the quadratic variation which we introduced earlier, we use the more robust quantity

$$
\left[V_{s} f\right](x, y)=\left[V_{s}^{(1)} f\right](x, y)=\sum_{t}\left|\left[W_{s} f_{t}\right](x, y)\right|
$$

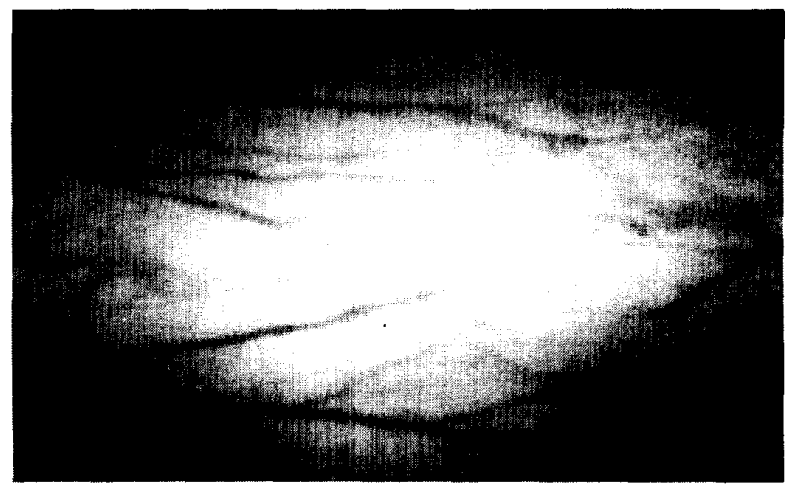

Fig. 5. Example of the use of the vibration map in the partial reconstruction of images. This image was reconstructed after removal from the original image at the top of the left column of Fig. 3, of all the local extrema nearby the secondary blood vessels as identified by the vibration map constructed as illustrated in Fig. 4. This procedure preserves the blood vessels that are stationary in time, but the vibration nearby the secondary blood vessels are removed. This phenomenon is very subtle and difficult to visualize: it is based on the time evolutions across several frames and it is of a lower scale than the main features of the images.

which captures the same features as $V_{s}^{(2)} f$. We then sum the variations over the resolutions to get the single image $V=\{V(x, y)\}_{(x, y)}$ defined by

$$
V(x, y)=\sum_{j=1}^{J}\left[V_{2^{j}} f\right](x, y) .
$$

- Next we compute the map of the high vibration points of the data. Given a threshold $\alpha$ between 0 and 1 , we compute the $100 \alpha$-th percentile, say $\pi_{\alpha}(V)$, of the $V$ values computed above. Next, we determine the pixels $(x, y)$ at which the total variation $V(x, y)$ is above this percentile threshold. In other words, we determine the set

$$
M=\left\{(x, y): V(x, y)>\pi_{\alpha}(V)\right\}
$$

which we call the vibration map.

- We now go back to the wavelet transforms $W_{s} f_{t}$ of the various time derivatives. We compute the maxima of the modulus of these transforms and we set to 0 all the maxima within a distance $\lambda s$ of a pixel of the vibration map $M$. Notice that the trimming of the extremas is done as a function of the locations of the extrema. This is in sharp contrast with the usual trimming procedures which are based on a thresholding depending only on the size of the extrema (see, for example, [4] and the discussion of the previous section illustrated by Fig. 3). After trimming, each time derivative image is reconstructed from the remaining extrema. In this way, we get a new sequence $\tilde{f}_{t}(1), \cdots, \tilde{f}_{t}\left(n_{f}-1\right)$ of images of the same sizes.

The images $\tilde{f}_{t}(\cdot)$ produced by the above algorithm are the same as the original images, except that most of the vibrations due to the active blood vessels have been removed.

\section{B. Intrinsic Signals and Active Regions}

We are now dealing with data which have been cleaned from the blood vessel vibrations. In other words, we can assume that the artifacts of the active blood vessel vibrations do not mask 

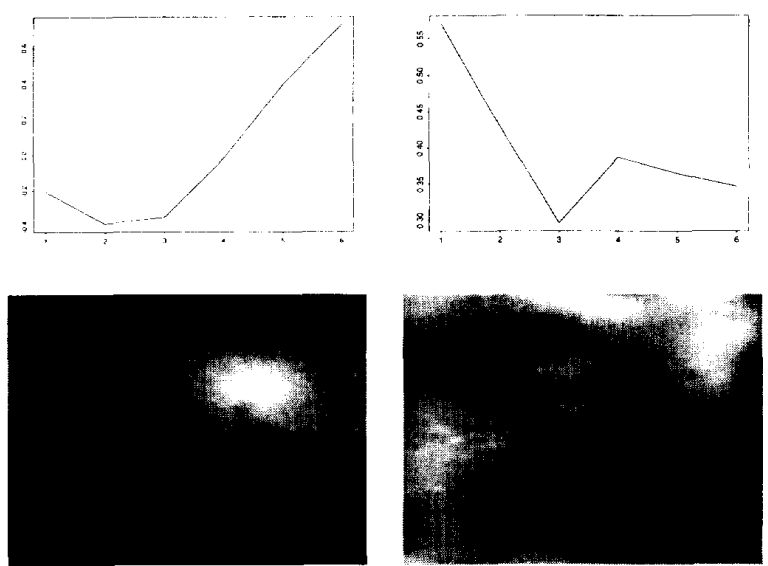

Fig. 6. (Left) Top: The time derivative of the intrinsic signal of the experiment considered throughout (recall Fig. 1 and the top part of Fig. 3), as given by the first normalized eigenvector after applying the PCA to the sequence of modified velocity images. Notice that the values are negative for the first part and positive after that. This plot is consistent with the model of intrinsic signal given by the function $N(t)$ (recall Fig. 2). Bottom: Image of the values of the sizes of the projections of the time evolutions of the modified velocities onto the first normalized eigenvector given in the top part. The region identified this way coincides with the area determined both anatomically and electrophysiologically. (Right) Top: The second normalized eigenvector given by the PCA. Notice that there is no change of sign. This type of time evolution does not correspond to the time evolution one expects (on biological grounds) from the active region. Bottom: Image of the sizes of the projections of time evolutions of the modified velocities onto the second eigenvector. These sizes are much smaller than those of the first projections. Scaling was performed to use the same gray scale. The information contained in this projection cannot be used. As earlier, we did not plot the first two values of the eigenvectors

the functional response to the stimuli. We finish the description of the algorithm with the steps involving the extraction of the intrinsic signals and the identification of the region where the response to the stimulus takes place.

As explained in the previous section, the main thrust of this last part of the algorithm is the use of principal component analysis (PCA) in the search for the characteristic "time evolutions" of the pixels in the active region. The covariance matrix is computed from $n_{r} \times n_{c}=144 \times 192$ observations. It is an $\left(n_{f}-1\right) \times\left(n_{f}-1\right)=8 \times 8$ matrix which is very easy to diagonalize. Recall the discussion in Section I to compare this use of the PCA with previous uses in similar imaging problems.

- Consider, at each pixel $(x, y)$, the modified time evolution of the response velocity $\tilde{f}_{t}(\cdot, x, y)$ as a vector in $n_{f}-1$ dimensions. Let $\left\{v^{(i)}(t)\right\}_{1 \leq t \leq n_{f}-1}$ be the $i$ th normalized eigenvector produced by the PCA. The first of these eigenvectors gives the typical time evolution of the response velocity of the points in the active region; see Figs. 6 and 7 for three different examples.

- For each pixel $(x, y)$, the number

$P^{(1)}(x, y)=\left\langle\tilde{f}_{t}(\cdot, x, y), v^{(1)}\right\rangle=\sum_{t=1}^{n_{f}-1} \tilde{f}_{t}(t, x, y) v^{(1)}(t)$

gives the size of the projection of the modified velocity time evolution of the pixel $(x, y)$ on to the first eigenvector. As explained in the previous section, it measures
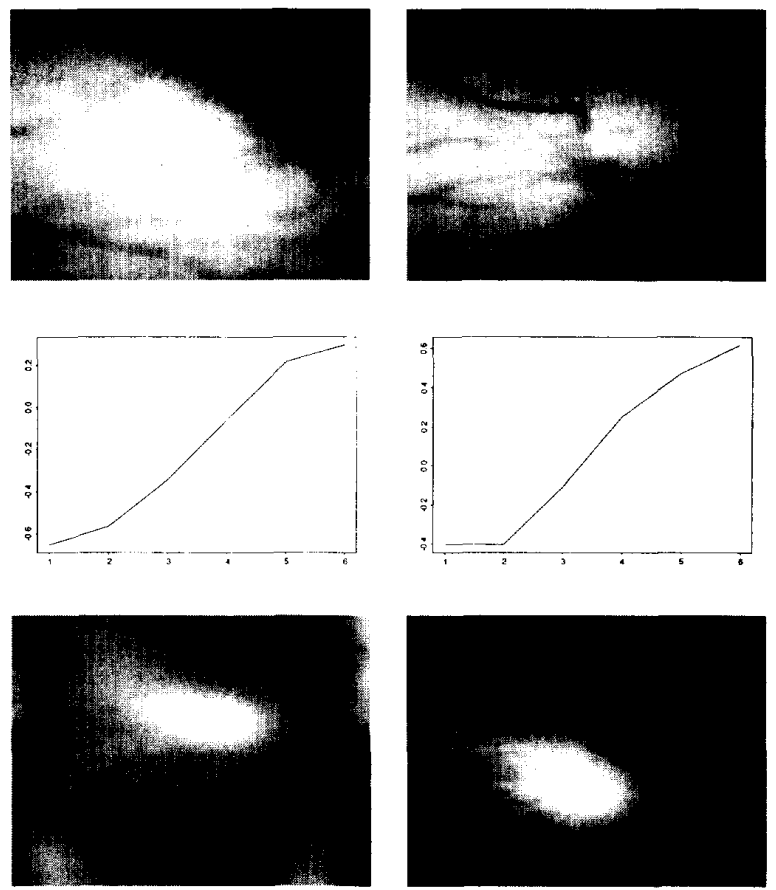

Fig. 7. (Left) Top: A typical image from another experiment. Middle: The intrinsic signal as identified by the first normalized eigenvector--notice the change of sign. Bottom: The corresponding activation region as identified by the values of the first eigen-projections. (Right) From top to bottom: Image from still another experiment, the first normalized eigenvector (for which the change in sign is noticed) and the corresponding activation region.

the degree of similarity with the prototype exemplified by the eigenvector $v^{(1)}$ in the sense that the larger $P^{(1)}(x, y)$, the more active the pixel. Imaging $\left\{p^{(1)}(x, y)\right\}_{(x, y)}$ gives a snapshot of the activity of the brain in response to the stimulus. Figs. 6 and 7 give three different examples of this projection. In each case, the high values are found in a compact region which we identify with the functional response to the stimulus. Fig. 6 also gives an illustration of the images produced by the second projection $P^{(2)}(x, y)$. No special region can be delineated in this case.

\section{EXPERIMENTAL RESULTS}

The algorithms described above have been implemented in the statistical data analysis environment Splus. (A Matlab implementation is currently being worked out by S. Zhong.) The images of five experiments conducted in the last six months (in the laboratory of R. D. Frostig) have been reanalyzed using the method described in this paper. The results of three of the experiments are reproduced in Figs. 6 and 7 (see [5] for more experimental results and comparisons with Fourier based analyses). Our results are consistent in the sense that:

- The first normalized eigenvector of the PCA of the changes in time of the modified velocities (i.e., of the images preprocessed to remove the main vibrations of the blood vessels) do change sign in the middle as expected. Recall the plot of $N(t)$ given in the bottom of Fig. 2 . 
- The second normalized PCA eigenvector DOES NOT change sign. It is either monotone increasing or monotone decreasing. This is an obvious contradiction with the type of time evolution examplified by our choice of the function $N(t)$. Recall that $N(t)$ (whose graph is given in the bottom of Fig. 2) is our mathematical model for the intrinsic signals.

- The image of the activity region as given by the size of the first eigen-projection is consistent with the results obtained previously with the various division methods and with the experimental verifications which followed.

These claims are illustrated in Fig. 6 in the case of the experimental data which we considered throughout the paper. Fig. 7 gives two examples of the precision and robustness of our method.

We close this section with a short discussion of the roles of the free parameters needed in the practical applications and the values we used in the experiments described in the paper. The first free parameter is the scale $s=2^{J}$ at which the wavelet transforms are computed. The resolution of the images (and consequently the size of the files to manipulate) was a factor in this choice. But it is fair to say that, in all the experiments we conducted, the properties of the images (dynamic range, Fourier localization, $\cdots$ ) were captured by these scales. The only choice left to the user concerns the vibration map. For the sake of generality, we introduced a parameter $\lambda$ in the definition of the size of the neighborhood of pixels to be included in the vibration map. This parameter is not of crucial importance: we used $\lambda=1$ in all the applications. The important parameter is the threshold used to trim the extrema of the wavelet transforms. We chose the 90\%-tile to discard extrema according to their size. This choice is reasonable for the data files we analyzed but it may need some adjustment in different situations.

\section{CONCLUSION}

We proved that it is possible to design and implement an algorithmic procedure to control the blood vessel artifacts and delineate the region of the brain where the functional activity occurs as a response to stimuli. Moreover, as a byproduct of the mathematical analysis, we get a realistic estimation of the actual time evolution of the light reflectance in the active region.

The reliability of the procedure and the crispness of the identification will prove to be crucial in the future use of this imaging technique. These procedures will be further applied to brain imaging, especially for imaging from chronic preparation before, during, and after manipulation to the system (such as drugs, changes in behavior, $\cdots$ ).

\section{ACKNOWLEDGMENT}

This work is part of a collaboration initiated within the Research Unit on Advanced Computing of the University of
California at Irvine. The authors would like to thank S. Zhong and the anonymous referees for their constructive comments.

\section{REFERENCES}

[1] E. Bartfeld and A. Grinvald, "Relationships between orientationpreference pinwheels, cytochrome oxydase blobs, and ocular dominance columns in primate striate cortex," in Proc. Nat. Acad. Sci., USA, vol. 89, pp. $11905-11909,1992$.

[2] J. R. Binder and S. M. Rao, "Human brain mapping with functional magnetic resonance imaging," in Localization and Neuroimaging in Neuropsychology. New York: Academic, 1994, pp. 185-212.

[3] T. Bonhoeffer and A. Grinvald, "Iso-orientation domains in the cat visual cortex are arranged in pinwheel-like patterns," Nature, vol. 353, pp. $429-431,1991$.

[4] R. Carmona, "Wavelet identification of transients in noisy signals," in Proc. SPIE, Math. Imag.: Wavelet Applicat. Signal Image Processing. San Diego, CA, June 15-16, 1993, pp. 392-400.

[5] R. Carmona, S. Zhong, C. H. Chen, and R. D. Frostig, "Brain-function imaging: A comparative study," 1995 (preprint).

[6] S. A. Engel, D. E. Rumelhart, B. A. Wandell, A. Lee, G. H. Glover, E. J. Chicilinsky, and M. N. Shalden, "fMRI of human visual cortex," Nature, vol. 369, p. 525, 1994.

[7] K. J. Friston, C. D. Frith, P. F. Liddle, and R. S. J. Frackowiack, "Functional connectivity: The principal component analysis of large (PET) data sets," J. Cerebral Blood Flow Metabolism, vol. 13, pp. 5-14, 1993.

[8] R. D. Frostig, E. E. Lieke, D. Y. Ts'o, and A. Grinvald, "Cortical functional architecture and local coupling between neuronal activity and the microcirculation revealed by in vivo high resolution optical imaging of intrinsic signals," in Proc. Nat. Acad. Sci., USA, vol. 87, pp. $6082-6086,1990$.

[9] A. Grinvald, E. E. Lieke, R. D. Frostig, C. D. Gilbert, and T. N. Wiesel, "Functional architecture of the cortex revealed by optical imaging of intrinsic signals," Nature, vol. 324, pp. 361-364, 1986.

[10] A. Kertesz, "Localization and function: Old issues revisited and new developments," in Localization and Neuroimaging in Neuropsychology. New York: Academic, 1994, pp. 1-34.

[11] K. K. Kwong, J. W. Belliveau, D. A. Chesler, I. E. Goldberg, R. M. Weisskoff, B. P. Poncelet, D. H. Kennedy, B. E. Hoppel, M. S. Cohen, R. Turner, H. M. Cheng, T. J. Brady, and B. Rosen, "Dynamic magnetic resonance imaging of human brain activity during primary sensory stimulation," in Proc. Nat. Acad. Sci, USA, vol. 89, pp. 5675-5679, 1992.

[12] S. Mallat and W. L. Hwang, "Singularities detection and processing with wavelets," IEEE Trans. Inform. Theory, vol. 38, no. 2, pp. 617-643, 1992.

[13] S. Mallat and S. Zhong, "Characterization of signals from multiscale edges," IEEE Trans. Pattern Anal. Machine Intell., vol. 14, pp. 710-732, 1992.

[14] S. A. Masino, M. C. Kwon, Y. Dori, and R. D. Frostig, "Characterization of functional organization within rat barrel cortex using intrinsic signal optical imaging through a thinned skull," in Proc. Nat. Acad. Sci., USA, vol. 90 , pp. $9998-10002,1993$.

[15] S. A. Masino, K. H. Tran, C. H. Chen, and R. D. Frostig, "Chronic optical imaging of intrinsic signals enables quantitative long term assessment of functional variability in rat barrel cortex," Soc. Neurosci., vol. 24, p. 122,1994

[16] S. Ogawa, D. W. Tank, R. Menon, J. M. Ellerman, S. G. Kim, $\mathrm{H}$. Merkle, and $\mathrm{K}$. Ugurbil, "Intrinsic signal change accompanying sensory simulation: Functional brain mapping with magnetic resonance imaging," in Proc. Nat. Acad. Sci., USA, vol. 89, pp. 5951-5955, 1992.

[17] L. Sirovich and R. Everson, "Management and analysis of large scientific data sets," Int. J. Supercomput. Applicat., vol. 6, pp. 50-68, 1992.

[18] D. Y. Ts'o. R. D. Frostig, E. E. Lieke, and A. Grinvald, "Functional organization in the primate visual cortex as revealed by optical imaging of intrinsic signals," Sci., vol. 249, pp, 417-420, 1990.

[19] S. Watanabe, Pattern Recognition: Human and Mechanical. New York: Wiley, 1985.

[20] E. Wilkinson, The Algebraic Eigenvalue Problem. Oxford: Clarendon, 1965. 